\title{
Confocal Fluorescence Microscope Studies of the Adsorptive Behavior of Dioctadecyl-rhodamine B Molecules at a Cyclohexane-Water Interface
}

\author{
Xue-Ying Zheng and Akira HaRata ${ }^{\dagger}$ \\ Department of Molecular and Material Sciences, Kyushu University, Kasuga, Fukuoka 816-8580, Japan
}

\begin{abstract}
The capabilities of using a confocal fluorescence microscope (CFM) for the interface-selective observation of dye molecules at an oil-water interface were evaluated. A simple expression for a criterion of interface selectivity was derived. The adsorptive behavior of dioctadecyl-rhodamine $\mathrm{B}$ at a cyclohexane-water interface was investigated experimentally to demonstrate the interface-selective observation with a CFM. The minimum number of detectable molecules in the probe area at the interface was estimated to be 82 for the present system. Preliminary experimental results of co-adsorption systems composed of adsorbed dye molecules and charged surfactants are also presented.
\end{abstract}

(Received September 29, 2000; Accepted October 25, 2000)

The liquid-liquid interface has been one of the most intriguing subjects of interest to scientists in fields as various as separation science, colloid chemistry, and biological chemistry. During the past two decades, much effort has been made to study this unique area mainly using surface-specific spectroscopic techniques. ${ }^{1-8}$ The advantage of employing these techniques to monitor a buried interface between two bulk phases is that there are no mechanical perturbation problems during the measurement. However, due to the limited sensitivity of these spectroscopic techniques, target molecules were usually studied in a relatively high surface-density region. With a high surface density, it is expected that the interaction between the adsorbed molecules, ${ }^{9}$ such as self-quenching from the highly fluorescent species to the non-fluorescent aggregates at the interface, ${ }^{10}$ can therefore be significant enough to affect the accuracy of detection. For a better understanding of the microscopic phenomenon and molecular behavior at the interface, it is important that highly sensitive approaches and techniques for the studies of probe molecules at a low surface-density region be developed.

A confocal fluorescence microscope (CFM) has the potential for such a highly sensitive measurement. ${ }^{11-14}$ With confocal excitation and detection, a femtoliter or subfemtoliter probe volume can be attained to largely suppress the background, which is the major source for any detection-sensitivity improvement. ${ }^{14}$ Although, intuitively, it would be difficult to apply CFM to study an interface, because it has poor depth resolution, which makes it difficult to clearly resolve the interface thickness, ${ }^{15}$ Zheng et al. have succeeded in using this technique to study insoluble as well as surface-active soluble chromphores at an air-water interface. ${ }^{16,17}$ For these two kinds of chromophores, owing to their preference of remaining at the interface, the bulk-originated fluorescence signal can be neglected and, thus, the depth disparity problem of the CFM can be resolved. For a liquid-liquid interface, since molecules can

$\doteqdot$ To whom correspondence should be addressed.

E-mail: harata@mm.kyushu-u.ac.jp possibly stay at either of the two bulk media and the interface, discriminating between the bulk- and the interface-originated signals is very complicated. However, with a careful selection of the probe chromophore, it is possible to apply a CFM for a liquid-liquid interface investigation.

Rhodamine dye is an amphiphilic molecule, which makes it a suitable chromophore for probing an interface. The chemical forms of rhodamine dyes depend on the polarity of the solvents: namely, because a non-fluorescent form dominates in non-polar or less-polar solvents, so does a fluorescent form dominate in polar solvents ${ }^{5}$ or at the interface. ${ }^{16,18}$ On the other hand, for an organic chromophore with long carbon chains, the distribution ratio in the water phase is negligible. ${ }^{19}$ Therefore, for a liquidliquid interface between water and a non-polar solvent (e.g., cyclohexane) containing an insoluble chromophore, such as dioctadecyl-rhodamine B ( $\mathrm{Rh}-\mathrm{C} 18)$, it is reasonable to suppose that only those dye molecules remaining at the interface contribute to the fluorescence signal.

In this work, an expression of the interface-selective criterion using a CFM was presented. By studying the adsorptive behavior of $\mathrm{Rh}-\mathrm{C} 18$ molecules at the interface between cyclohexane and water, we experimentally demonstrated the capabilities of using a CFM for interface-selective observations at the liquid-liquid interface. The minimum detectable molecular density for the present system was further estimated, and assumptions required for the estimation are discussed. Preliminary experimental results concerning the influence of charged surfactants on the adsorption of Rh-C18 to the interface are also presented.

\section{Experimental}

The experimental set-up of the CFM is similar to that reported previously. ${ }^{12,16}$ A 514.5-nm argon-ion laser beam was tuned to pass through a laser-line pass filter (515FS10-12.5, Andover Co.) and then focused by a lens before coupling to a microscope (Nikon labophot-2) with a pellicle beamsplitter (44-4059, Coherent, Auburn Group). Inside the microscope, the laser 
beam was focused to a spot with a radius of $0.66 \mu \mathrm{m}$ by a $10 \times$ objective lens with a numerical aperture of 0.25 (Nikon, Japan). Fluorescence originating from the focused region was collected by the same objective lens and then passed through the beamsplitter and an interference long-pass filter (550 FG 05-25, Andover Co.) to cut off the laser light and the scattering. After focusing on a pinhole (diameter, $200 \mu \mathrm{m}$ ) to eliminate out-offocus signals, the residual fluorescence was detected with a photomultiplier tube (R7400, Hamamatsu, Japan) and the signal was recorded in a photon-counting mode. The time period of each data record was $10 \mathrm{~s}$ with a $0.5-\mathrm{ms}$ integration time. An IBM-compatible computer was used for data analysis.

For CFM detection at the oil-liquid interface, the procedures used in the fluorescence measurement can be summarized as follows. A 5-mL Rh-C18 cyclohexane solution was pipetted to a water solution $(5 \mathrm{ml})$ contained in a cylindrical quartz cell (diameter $5 \mathrm{~cm}$ ). The sample was then placed on the stage of a microscope for about $20 \mathrm{~min}$ prior to detection. Finally, the satellite lines of the excitation laser were used as an inherent reference to adjust the focus point at the oil-water interface during the measurement. ${ }^{20}$

The $\mathrm{Rh}-\mathrm{C} 18$ working solution was prepared by serially diluting a $5.0 \times 10^{-4} \mathrm{M}$ Rh-C18 ethanol stock solution. The fluorescence dye, Rh-C18, was purchased from Molecular Probes, Inc., and cyclohexane was obtained from the Kishida Chemical Co., Ltd. Two kinds of surfactants were employed: sodium dodecylsulfate (SDS, Kishida Chemical Co., Ltd.) and cetyldimethylethylammonium bromide (CDMEAB, Tokyo Kasei Kogyo Co., Ltd.). All chemicals were of analytical grade and were used as received. Water was doubly deionized by a Milli-Q system (Millipore S. A., Molshem, France). All measurements were performed at $18 \pm 1^{\circ} \mathrm{C}$.

\section{Results and Discussion}

Selective fluorescence detection from an oil-water interface with a CFM

In a confocal fluorescence microscope, the probe volume is effectively confined to be an elongated cylinder ${ }^{12,16}$ with a radius of $\omega$ and a height of $2 d_{z}$. If the position of an oil-water interface is defined to be exactly at the symmetrical plane horizontally intersecting the cylinder, the probe area and probe volume are $\pi \omega^{2}$ and $2 \pi \omega^{2} d_{z}$ when the interface is observed (interface observation), while they are zero and $2 \pi \omega^{2} d_{\mathrm{z}}$ for a bulk observation, respectively. With a time-averaged measurement, the minimum ratio $\left(R_{\mathrm{f}}^{\mathrm{min}}\right)$ of the fluorescent photon number detected by an interface observation with respect to a bulk observation can be given as

$$
\begin{aligned}
R_{\mathrm{f}}^{\min }= & \left(\eta_{\mathrm{s}} \pi \omega^{2} N_{\mathrm{s}}+\eta_{\mathrm{o}} \pi \omega^{2} d_{\mathrm{z}} C_{\mathrm{o}}+\eta_{\mathrm{w}} \pi \omega^{2} d_{\mathrm{z}} C_{\mathrm{w}}\right) / \max \\
& \left(2 \eta_{\mathrm{o}} \pi \omega^{2} d_{\mathrm{z}} C_{\mathrm{o}}, 2 \eta_{\mathrm{w}} \pi \omega^{2} d_{\mathrm{z}} C_{\mathrm{w}}\right) \\
= & \left(\eta_{\mathrm{s}} N_{\mathrm{s}} / d_{\mathrm{z}}+\eta_{\mathrm{o}} C_{\mathrm{o}}+\eta_{\mathrm{w}} C_{\mathrm{w}}\right) / 2 \max \left(\eta_{\mathrm{o}} C_{\mathrm{o}}, \eta_{\mathrm{w}} C_{\mathrm{w}}\right),
\end{aligned}
$$

where $\eta_{\mathrm{s}}, \eta_{\mathrm{o}}$, and $\eta_{\mathrm{w}}$ represent the efficiencies of the fluorescence excitation-detection for fluorescent molecules at the interface and in the oil and water phases, respectively; $N_{\mathrm{s}}$ is the molecular density of dyes at the interface; and $C_{\mathrm{o}}$ and $C_{\mathrm{w}}$ are the bulk concentrations in the oil and water phases, respectively. The operator $\max (x, y)=x$ when $x>y$ and vice versa. We propose the value of $R_{\mathrm{f}}^{\text {min }}$ to be a criterion for an interfaceselective fluorescence observation with a CFM, because $R_{\mathrm{f}}^{\min } \gg 1$ means that the fluorescence signal from bulk solutions is negligible and also due to its simple form.

When a bulk solution has a low concentration at equilibrium

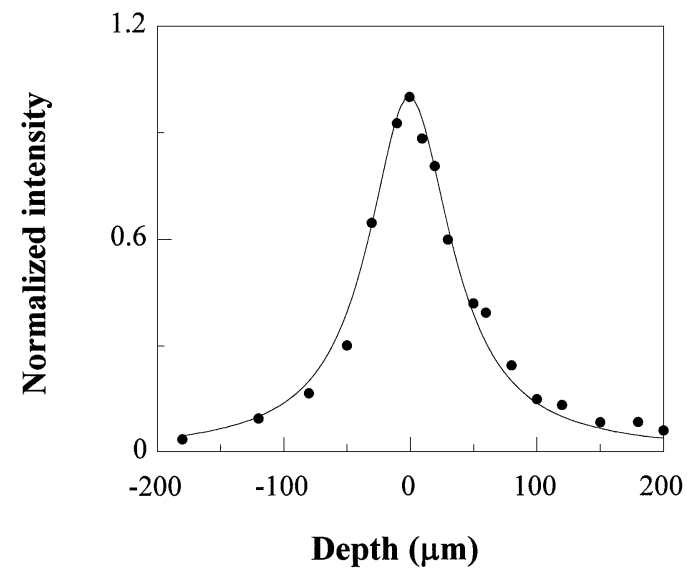

Fig. 1 Depth resolution of a confocal fluorescence microscope with a $10 \times$ objective. The numerical aperture of the objective is 0.25 . The position with the maximum intensity at the interface is set at 0 in the abscissa, of which the intensity is regarded as a unit. The minus values represent the upper positions in the oil phase, while the positive values represent those positions below the interface (water phase). The points $(\bullet)$ are the experimental data, and the solid line serves as a guide for the eyes.

we can assume $N_{\mathrm{s}}=L_{\mathrm{w}} C_{\mathrm{w}},=L_{\mathrm{o}} C_{\mathrm{o}}$, where $L_{\mathrm{w}}=K_{\mathrm{w}} N_{\max }$ and $L_{\mathrm{o}}=$ $K_{\mathrm{o}} N_{\max }$. Here, $K_{\mathrm{w}}$ and $K_{\mathrm{o}}$ are adsorption equilibrium constants for the target molecules in the water and oil phases, respectively, and $N_{\max }$ is the maximum surface density of the target molecules at the interface. As aforementioned, by using the CFM to study dye molecules at the interface, a careful selection of the chromophore is necessary. Usually, an oilsoluble but water-insoluble, or a water-soluble but oil-insoluble chromophore is selected. In this case, Eq. (1) can be reduced to

$$
R_{\mathrm{f}}^{\min }=0.5\left\{\min \left[\left(\eta_{\mathrm{s}} / \eta_{\mathrm{o}}\right) L_{\mathrm{o}},\left(\eta_{\mathrm{s}} / \eta_{\mathrm{w}}\right) L_{\mathrm{w}}\right] / d_{\mathrm{z}}+1\right\},
$$

where the operator $\min (x, y)=x$ when $x<y$ and vice versa. The values of $\eta_{\mathrm{s}}, \eta_{\mathrm{o}}, \eta_{\mathrm{w}}, L_{\mathrm{o}}$, and $L_{\mathrm{w}}$ are intrinsic properties of the fluorescent dyes under certain conditions. It can thus be said that the depth resolution of CFM indexed by $d_{z}$ plays a decisive role in determining the value of $R_{\mathrm{f}}^{\text {min }}$.

In order to evaluate the depth resolution and to testify the interface-selective observation with the CFM of Rh-C18 molecules at the cyclohexane-water interface, a fluorescence signal was observed as a function of the depth position. The result is shown in Fig. 1, in which the concentration of an RhC18 cyclohexane solution is $5 \times 10^{-10} \mathrm{M}$, and the signal intensity is normalized. The position with a maximum fluorescence signal is regarded as the position of the interface. The signal in Fig. 1 has $80 \mu \mathrm{m}$ of the full width at half maximum (FWHM), corresponding to $d_{\mathrm{z}}=40 \mu \mathrm{m}$. In addition, the signal from either of the bulk media was almost at the background level. From this depth resolution study, we can easily move the stage upwards or downwards to carry out interface and bulk observations and, thus, the value of $R_{\mathrm{f}}^{\text {min }}$ can be determined experimentally from these two types of measurements. According to the definition of $R_{\mathrm{f}}^{\min }, R_{\mathrm{f}}^{\mathrm{min}}=14$ could be obtained by using the experimental data shown in Fig. 1. These results confirm that an interface-selective observation of Rh-C18 molecules could be performed with a CFM, and $R_{\mathrm{f}}^{\text {min }}$ could be regarded as a criterion to evaluate the surface selectivity. It is known that $d_{\mathrm{z}}$ is determined by the numerical aperture of the microscope objective. ${ }^{12}$ Decreasing the size of 


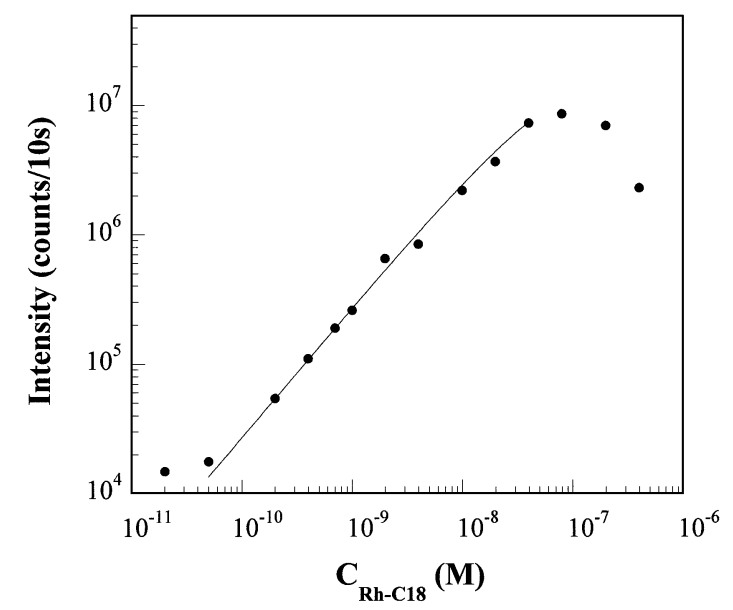

Fig. 2 Fluorescence intensity as a function of the concentration of the Rh-C18 cyclohexane solution. The points (•) stand for the experimental data, and the solid line represents the fitting curve calculated from the Langmuir equation.

the pinhole and/or increasing the numerical aperture of the objective can correspondingly reduce the $d_{\mathrm{z}},{ }^{21}$ and, thus, enlarge the $R_{\mathrm{f}}^{\mathrm{min}}$. This indicates that the CFM has a great potential for improving the interface-selective observation.

\section{Estimation of the dye molecular density at a cyclohexane-water} interface

When a few molecules of fluorescent substance pass across the probe area focused by a CFM at the interface, fluorescence photon bursts (time-dependent fluctuation of the photon counts) are expected to be observed as those detected from dye molecules at an air-water interface. ${ }^{16,17}$ However, we observed no fluorescence photon bursts under the present experimental conditions, which might be due to the small numerical aperture of the objective lens that was used and/or the large number of observed molecules at the probe area. In addition, as aforementioned, the lower surface density can by and large reduce the intermolecular interaction. Thus, it is important to evaluate the minimum number of dye molecules detectable with the CFM, which we used.

If the self-quenching and energy transfer between adsorbed dye molecules are negligible, the time-averaged fluorescence signal $\left(I_{\mathrm{F}}\right)($ counts/s, for $10 \mathrm{~s})$ is proportional to the mean number of dye molecules in the probe area, given as a product of the surface density $N_{\mathrm{s}}$ (molecules $/ \mathrm{cm}^{2}$ ) and the probe area $A_{\mathrm{p}}$ $\left(=\pi \omega^{2}=1.4 \times 10^{-8} \mathrm{~cm}^{2}\right)$ at the interface,

$$
I_{\mathrm{F}}=G N_{\mathrm{s}} A_{\mathrm{p}},
$$

where $G$ (counts/molecule, in $10 \mathrm{~s}$ ) is a proportional constant representing the number of photon counts detected per molecule in $10 \mathrm{~s}$. If the adsorption process of Rh-C18 molecules to the interface is described by a reaction between $\mathrm{Rh}-\mathrm{C} 18$ molecules in the cyclohexane phase and the interface, ${ }^{22,23}$ we can assume an adsorption isotherm (concentration dependence of surface density) represented by

$$
N_{\mathrm{s}}=N_{\max } K_{\mathrm{o}} C_{\mathrm{o}} /\left(1+K_{\mathrm{o}} C_{\mathrm{o}}\right) .
$$

Equation (4) can be derived with the same assumptions from the Langmuir isotherm. At a low concentration, Eq. (4) can be deduced to $N_{\mathrm{s}}=L_{\mathrm{o}} C_{\mathrm{o}}$. The reason that $C_{\mathrm{o}}$, rather than $C_{\mathrm{w}}$, was used is that Rh-C18 is a water-insoluble, but an oil-soluble molecule, having a negligible $C_{\mathrm{w}}$ and a measurable $C_{\mathrm{o}}$.

The adsorption isotherm of Rh-C18 molecules to the cyclohexane-water interface was investigated by measuring the fluorescence intensity for an interface with various concentrations of Rh-C18 cyclohexane solutions. The result is shown in Fig. 2, in which a $5 \mathrm{~mL}$ volume of a Rh-C18 solution with a concentration ranging from $5 \times 10^{-11} \mathrm{M}$ to $4 \times 10^{-7} \mathrm{M}$ was added to the surface of the water phase, and the fluorescence intensity was expressed as the accumulated counts in $10 \mathrm{~s}$. When the concentration is low, the fluorescence signal is proportional to the $\mathrm{Rh}-\mathrm{C} 18$ concentration, indicating that the number of adsorbed molecules increases linearly with the concentration. At a medium concentration of around $10^{-8} \mathrm{M}$, the signal behaves as if it were saturated, and after reaching a maximum fluorescence intensity at about $8 \times 10^{-8} \mathrm{M}$, the fluorescence signal decreases when the Rh-C18 concentration is further increased. Such fluorescence saturation can be due to adsorption saturation, and the signal decrease can be attributed to a quenching by an intermolecular energy transfer. ${ }^{5}$

By fitting the fluorescence intensity versus the concentration with Eq. (4), and then combining Eq. (3), the values of $K_{\mathrm{o}}$ and $G N_{\max }$ were obtained to be $1.2 \times 10^{7} \mathrm{M}^{-1}$ and $2.3 \times 10^{7}$ counts $/ 10$ $\mathrm{s}$, respectively. The fitting process was conducted when the concentration was below $4 \times 10^{-8} \mathrm{M}$ in order to reduce the influence of fluorescence quenching by intermolecular interaction as much as possible. The Gibbs energy of adsorption, $\left(-\Delta G_{\mathrm{ads}}{ }^{\circ}\right)$ corresponding to the adsorption constant, $\left(K_{\mathrm{o}}\right)$ was further calculated to be $39 \mathrm{~kJ} / \mathrm{mol}$ with the relation $\Delta G_{\text {ads }}{ }^{\circ}=-R T \ln K_{0}$, where $R$ is the gas constant and $T$ is the absolute temperature $(291 \mathrm{~K}){ }^{8}$ This value is comparable to the measured adsorption energy for other amphiphiles adsorbing from the organic phase to the liquid-liquid interface. ${ }^{24}$

The maximum surface density $\left(N_{\max }\right)$ cannot be deduced from the experimental data directly, because it is unable to determine the fluorescence signal corresponding to the full surface coverage due to self-quenching. From the surface-pressure isotherm ( $\Pi-A$ curve) of an Rh-C18 monolayer compressed by the Langmuir-Blodgett technique on the water surface, ${ }^{25}$ the average area-per-molecule value is about $210 \AA^{2}$, corresponding to a surface density of $\sim 8 \times 10^{-11} \mathrm{~mol} / \mathrm{cm}^{2}$. It is known that a repulsive interaction might prevent adsorbed species from packing tightly at the liquid-liquid interface, resulting in occupying a larger average area per molecule. ${ }^{9}$ In this work, however, the maximum surface density of $\mathrm{Rh}-\mathrm{C} 18$ molecules at the liquid-liquid interface was assumed to be $8 \times 10^{-11} \mathrm{~mol} / \mathrm{cm}^{2}$.

There remains a problem that the initial concentration $\left(C_{\text {ini }}\right)$, rather than the equilibrium concentration $\left(C_{\mathrm{o}}\right)$, was used in the fitting procedure. $C_{\mathrm{o}}$ should be different from $C_{\mathrm{ini}}$, because of the adsorption of Rh-C18 to the interface. Based on the mass balance, $C_{\mathrm{o}}$ can be related to $C_{\mathrm{ini}}$ as the following equation:

$$
C_{\text {ini }} V=C_{\mathrm{o}} V+N_{\mathrm{s}} A,
$$

where $V$ is the volume of an $\mathrm{Rh}-\mathrm{C} 18$ cyclohexane solution $(5 \times$ $\left.10^{-3} \mathrm{~L}\right)$, and $A$ is the total area of the interface $\left(19.6 \mathrm{~cm}^{2}\right)$.

At a low coverage, combining Eq. (5) with $N_{\mathrm{s}}=N_{\max } K_{\mathrm{o}} C_{\mathrm{o}}$, we can get $C_{\mathrm{o}}=0.2 C_{\text {ini }}$. When a $5 \times 10^{-11} \mathrm{M}$ Rh-C18 cyclohexane solution is considered, the surface density is estimated to be $1 \times$ $10^{-14} \mathrm{~mol} / \mathrm{cm}^{2}$, corresponding to 82 molecules in the probe area at the interface $\left(1.4 \mu \mathrm{m}^{2}\right)$.

Interaction of Rh-C18 with ionic surfactants at the oil-water interface

A preliminary result of an investigation of the molecular 


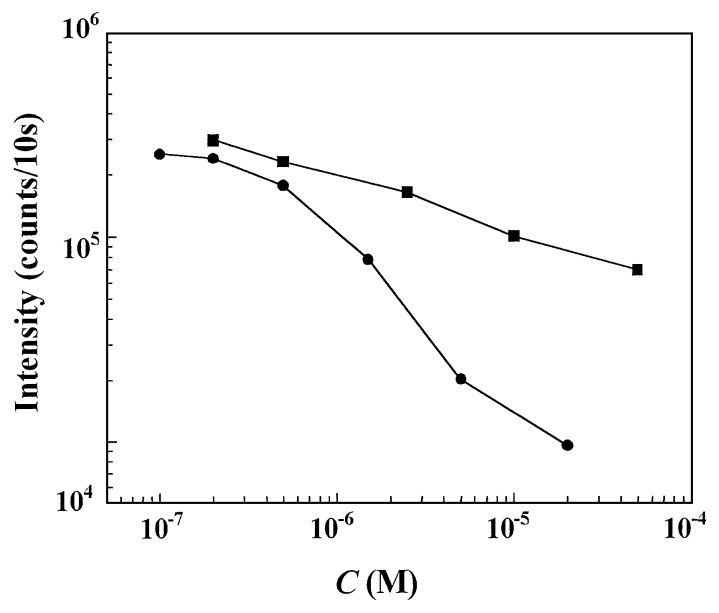

Fig. 3 Dependence of fluorescence intensity on the concentration of a positively charged surfactant $(\mathrm{CDMEAB}, \bullet)$ and a negatively charged surfactant (SDS, - ) at the water phase. The initial concentration of the Rh-C18 cyclohexane solution is $5 \times 10^{-9} \mathrm{M}$. The solid line is provided for a clear reading.

interaction at the oil-water interface with a CFM is presented. The Rh-C18 molecule at the interface is in zwitterionic and cationic forms, ${ }^{26}$ rather than in the lactone form. Therefore, it would be interesting to study the interaction between the chromophore and charged surfactants at the interface. In this work, CDMEAB and SDS were used as positively and negatively charged surfactants, respectively. The fluorescence signal from the interface-adsorbed Rh-C18 molecules was observed as a function of the bulk concentration of surfactants, in which the concentration of an Rh-C18 cyclohexane solution was $5 \times 10^{-9} \mathrm{M}$ (Fig. 3). It can be seen that the fluorescence signal reached a maximum value in the absence of surfactants, while a large decrease, depending on the surfactant concentration, was observed in the presence of either CDMEAB or SDS, regardless of the polarity of the surfactant charge. This result indicates that electrostatic forces do not dominate the interaction between these charged surfactants and the chromophore. A competitive adsorption mechanism at the interface is considered to be the origin of the signal decrease. This mechanism is different from the case of multipoint electrostatic coadsorption between surfactants and a biopolymer, where the formation of a bilayer is introduced. ${ }^{9,27}$ It is noticeable from Fig. 3 that the signal decrease induced by SDS is less than that by CDMEAB. This result can be explained as the coexistence of zwitterionic and cationic chromophores at the interface, resulting in a relatively smaller repulsive interaction between the SDS molecules and the chromophore, given that the adsorption strength and the maximum surface coverage for both CDMEAB and SDS are comparable. Further studies, in which the sensitivity increase will play an essential role in understanding, are required for details.

In this work, we showed that a confocal fluorescence microscope has a good potential for interface-selective observations of dye molecules at oil-water interfaces, which was confirmed by studying the adsorptive behavior of dioctadecyl-rhodamine B at an interface between cyclohexane and water. A criterion of interface selectivity with a simple expression has been proposed. The minimum number of detectable molecules in the probe area was estimated to be 82 for the present system based on some assumptions. Preliminary experimental results of co-adsorption systems were also presented.

Since a certain protein or nucleotide could be labeled with more than 80 fluorescent chromophores, a single molecule detection for such large molecules may be possible at an oilwater interface, even at the present status of the CFM. Furthermore, there is much room for improvements of the sensitivity and surface selectivity. An investigation of single fluorescent molecules at the oil-water interface would provide valuable information, especially for environmental and biological science. We assume that analytical chemistry and interface science based on single molecule detection at interfaces will be a promising scientific field in the future.

\section{Acknowledgements}

The authors are very appreciative of Dr. Y. Hatano of Kyushu University for his valuable participation in discussion. This work is financially supported by a Grant-in-Aid for Scientific Research (No. 12875161) from the Japanese Ministry of Education, Science, Sports, and Culture.

\section{References}

1. R. M. Corn and D. A. Higgins, Chem. Rev., 1994, 94, 107, and references therein.

2. R. R. Naujok, H. J. Paul, and R. M. Corn, J. Phys. Chem. A, 1996, 100, 10497.

3. M. C. Messmer, J. C. Conboy, and G. L. Richmond, J. Am. Chem. Soc., 1995, 117, 8039.

4. P. B. Miranda and Y. R. Shen, J. Phys. Chem. B, 1999, 103, 3293.

5. H. Watarai and F. Funaki, Langmuir, 1996, 12, 6717.

6. D. A. Piasecki and M. J. Wirth, J. Phys. Chem., 1993, 97, 7700 .

7. Z. H. Zhang, I. Tsuyumoto, T. Kitamori, and T. Sawada, J. Phys. Chem. B, 1998, 102, 10284.

8. Z. H. Zhang, I. Tsuyumoto, S. Takahashi, T. Kitamori, and T. Sawada, J. Phys. Chem. A, 1997, 101, 4163.

9. H. J. Paul and R. M. Corn, J. Phys. Chem. B, 1997, 101, 4494.

10. A. K. Dutta and C. Salesse, Langmuir, 1997, 13, 5401.

11. M. Eigen and R. Rigler, Proc. Natl. Acad. Sci. USA, 1994, 91,5740 .

12. S. Nie, D. T. Chiu, and R. N. Zare, Anal. Chem., 1995, 67, 2849.

13. T. Schmidt, G. J. Schütz, W. Baumgartner, H. J. Gruber, and H. Schindler, J. Phys. Chem., 1995, 99, 17662.

14. W. P. Ambrose, P. M., Goodwin, J. H. Jett, A. V. Orden, J. H. Werner, and R. A. Keller, Chem. Rev., 1999, 99, 2929.

15. Y.-Q. Li, S. Sasaki, T. Inoue, and T. Ogawa, Laser Chem., 1998, 17, 175.

16. X.-Y. Zheng, A. Harata, and T. Ogawa, Chem. Phys. Lett., 2000, 316, 6 .

17. X.-Y. Zheng, A. Harata, and T. Ogawa, Spectrochim. Acta: Part $A$, in press.

18. M. N. Slyadnev, T. Inoue, A. Harata, and T. Ogawa, Colloids Surf. A, 2000, 164, 155.

19. X. Zhao, S. Subrahmanyan, and K. B. Eisenthal, Chem. Phys. Lett., 1990, 171, 558.

20. Y.-Q. Li, T. Inoue, A. Harata, and T. Ogawa, Instru. Sci. Tech., 1999, 27, 159. 
21. J. H. Massig and T. Hellmuth, Optik, 1993, 95, 86.

22. A. Castro, K. Bhattacharyya, and K. B. Eisenthal, J. Chem. Phys., 1991, 95, 1310.

23. R. L. Hansen and J. M. Harris, Anal. Chem., 1998, 70 , 2565.

24. D. A. Higgins and R. M. Corn, J. Phys. Chem., 1993, 97 , 489.
25. O. N. Slyadneva, M. N. Slyadneva, V. M. Tsukanova, T. Inoue, A. Harata, and T. Ogawa, Langmuir, 1999, 15, 8651.

26. I. L. Arbeloa and K. K. Rohatgi-Mukherjee, Chem. Phys. Lett., 1986, 128, 474.

27. J. Rinuy, P. F. Brevet, and H. H. Girault, Biophys. J., 1999 , 77,3350 . 